\title{
Utilisation du charbon de bois comme principale source d'énergie de la population urbaine : un sérieux problème pour la conservation du couvert forestier au Burundi
}

Frédéric BANGIRINAMA ${ }^{1}$

Bonaventure NZITWANAYO ${ }^{2}$

Paul HAKIZIMANA ${ }^{2}$

${ }^{1}$ École normale supérieure

Département des Sciences naturelles

BP 6983, Bujumbura

Burundi

${ }^{2}$ Université du Burundi

Faculté des Sciences

BP 2700, Bujumbura

Burundi

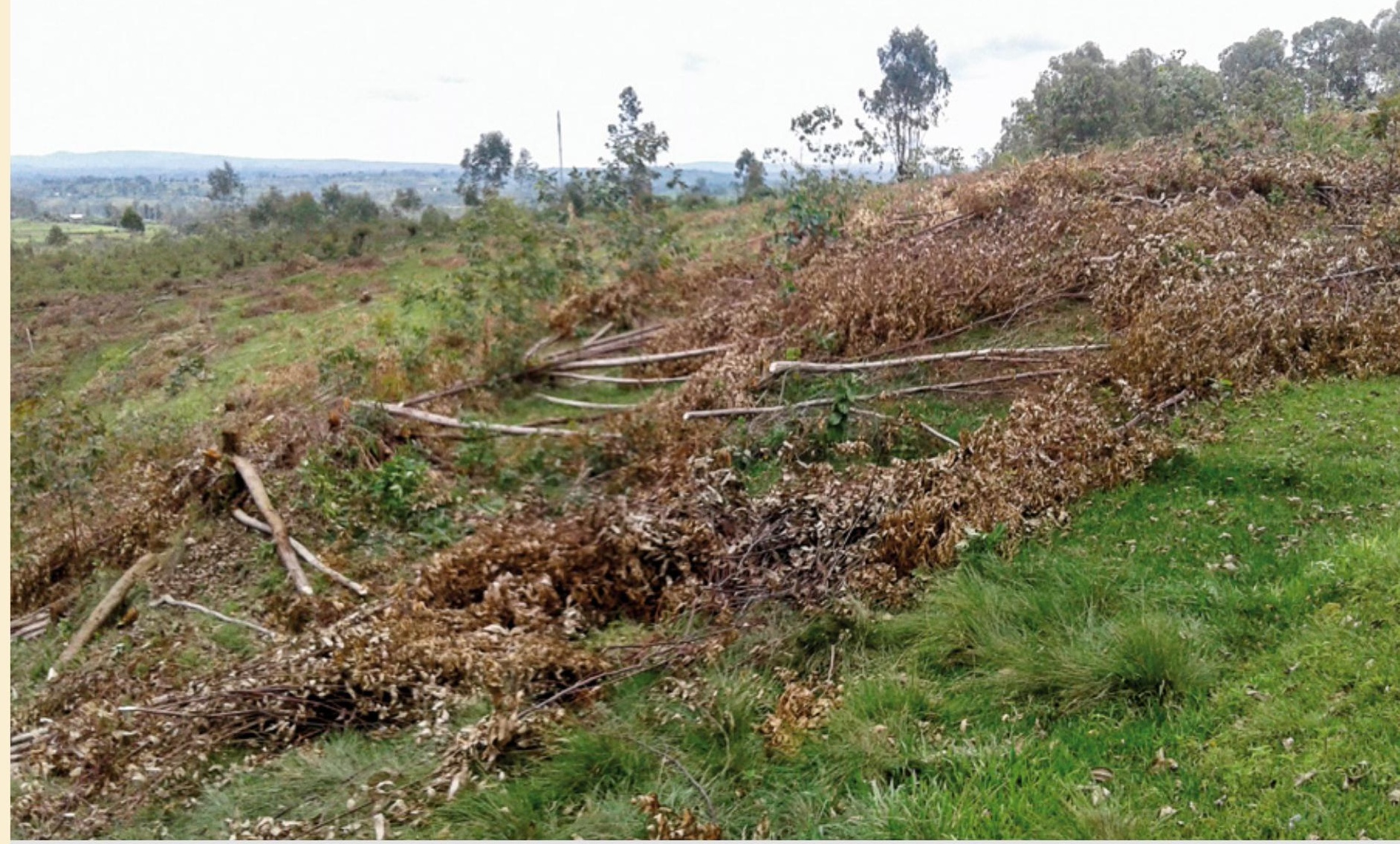

Photo 1.

Coupe rase d'eucalyptus pour la carbonisation (commune de Buraza dans la province de Gitega).

Photo F. Bangirinama. 


\section{RÉSUMÉ}

\section{UTILISATION DU CHARBON DE BOIS COMME PRINCIPALE SOURCE D'ÉNERGIE DE LA POPULATION URBAINE : UN SÉRIEUX PROBLĖME POUR LA CONSERVATION DU COUVERT FORESTIER AU BURUNDI}

La question de l'énergie domestique reste une préoccupation majeure dans le monde, en particulier dans les pays en développement où la majorité de la population utilise le bois de chauffage, le charbon de bois et les résidus agricoles pour satisfaire leurs besoins énergétiques liés à la cuisson des aliments. Au Burundi, où le bois constitue la principale source d'énergie domestique avec $96,6 \%$ du bilan énergétique global, le charbon de bois est consommé à $77 \%$ par la population urbaine. Selon une enquête menée sur les grands axes routiers, 56548 tonnes et 13552 tonnes de charbon de bois sont respectivement consommées par la population urbaine de Bujumbura et Gitega, soit un total de 70100 tonnes. La consommation annuelle estimée s'élève à 104718 tonnes de charbon de bois pour la seule population urbaine. Cette consommation par la population de Gitega et Bujumbura entraîne une perte annuelle de 3505 à 4673 ha de couvert forestier, soit annuellement entre 5236 et 6980 ha si l'on extrapole ces chiffres à l'ensemble de la population urbaine du Burundi. À ce rythme, le couvert forestier du Burundi, estimé à 171625 ha, pourrait disparaître dans 25 à 33 ans. Pour garantir la durabilité du système d'approvisionnement énergétique de la population urbaine burundaise, de nouvelles techniques et pratiques doivent être adoptées, comme la plantation d'arbres le long des axes routiers, la régénération naturelle assistée, la recherche d'espèces et variétés mieux adaptées et plus rentables, le recours aux énergies renouvelables, la valorisation énergétique des déchets et l'amélioration de l'efficience énergétique (foyers améliorés, par exemple).

Mots-clés : énergie domestique, charbon de bois, déforestation, urbanisation, gestion durable, Burundi.

\section{ABSTRACT}

\section{CHARCOAL AS THE MAIN SOURCE OF FUEL FOR CITY DWELLERS: A SERIOUS PROBLEM FOR THE CONSERVATION OF BURUNDI'S FOREST COVER}

The issue of domestic energy remains a major concern worldwide and particularly in developing countries, where the majority of the population still uses firewood, charcoal and agricultural residues as fuel for cooking. In Burundi, where wood is the main source of domestic energy and accounts for $96.6 \%$ of total energy use, $77 \%$ of all charcoal supplies are used by city dwellers. The results of a survey conducted along major roads show that the urban populations of Bujumbura and Gitega consume a total of 70,100 tonnes of charcoal (56,548 and 13,552 tonnes respectively). Annual charcoal consumption by the country's entire urban population was estimated in our study at 104718 tonnes. Charcoal consumption in Gitega and Bujumbura alone destroys 3,505-4,673 ha of forest cover per year, with the figure for Burundi's total urban population reaching 5,236 to 6,980 ha. At this rate, Burundi's entire forest cover, currently estimated at 171,625 ha, will disappear in about 25 to 33 years. For the system to be sustainable, new techniques and practices must be adopted, such as planting trees along roads, assisted natural regeneration, research on the most suitable and productive species or varieties, increased use of renewable energy, waste-to-energy techniques and energy efficiency improvements (e.g. improved stoves).

Keywords: domestic energy, charcoal, deforestation, urbanisation, sustainability, Burundi.

\section{RESUMEN}

USO DEL CARBÓN VEGETAL COMO PRINCIPAL FUENTE DE ENERGÍA POR LA POBLACIÓN URBANA: UN SERIO PROBLEMA PARA LA CONSERVACIÓN DE LA CUBIERTA FORESTAL EN BURUNDI

La cuestión de la energía doméstica sigue siendo motivo de gran preocupación en todo el mundo, especialmente en los países en vías de desarrollo donde la mayoría de la población usa leña, carbón vegetal y residuos agrícolas para satisfacer sus necesidades energéticas ligadas a la cocción de alimentos. En Burundi la madera supone la principal fuente de energía doméstica con el 96,6\% del balance energético global. El $77 \%$ del carbón vegetal lo consume la población urbana. Una encuesta realizada en los principales ejes viales muestra que la población urbana de Bujumbura y Gitega consume 56548 y 13552 toneladas de carbón respectivamente, o sea, un total de 70100 toneladas. Contabilizando sólo a la población urbana, el consumo anual estimado asciende a 104718 toneladas de carbón vegetal. Sólo el consumo de los habitantes de Gitega y Bujumbura ya representa una pérdida anual de 3505 a 4673 ha de cubierta forestal y, extrapolando estas cifras al conjunto de la población urbana de Burundi, obtenemos una pérdida anual de 5236 a 6980 ha. A este ritmo, la cubierta forestal de Burundi, estimada en 171625 ha, podría desaparecer en unos 25 a 33 años. Para garantizar la sostenibilidad del sistema de abastecimiento energético de la población urbana de Burundi, hay que incorporar nuevas técnicas y prácticas como la siembra de árboles a lo largo de los ejes viales, la regeneración natural asistida, la búsqueda de especies y variedades mejor adaptadas y más rentables, el uso de energías renovables, la valorización energética de los residuos y la mejora de la eficiencia energética ( $p$. ej. cocinas mejoradas).

Palabras clave: energía doméstica, carbón vegetal, deforestación, urbanización, manejo sostenible, Burundi. 


\section{Introduction}

La question de l'énergie domestique reste une préoccupation majeure dans le monde et en particulier dans les pays en développement, où la majorité de la population utilise essentiellement le bois de chauffage, le charbon de bois et les résidus agricoles pour satisfaire ses besoins énergétiques liés à la cuisson des aliments (Aruna Sefu, 2011).

Dans la sous-région de la Communauté économique des pays des Grands Lacs, le bois et les produits lignocellulosiques représentent plus de $97 \%$ du bilan énergétique. Plus de $90 \%$ de la population des villes et des centres urbains secondaires utilisent le charbon de bois comme source principale d'énergie domestique, surtout pour la cuisson des aliments (MEM, 2011a).

$\mathrm{Au}$ Burundi, les combustibles traditionnels (bois, charbon de bois, résidus agricoles) couvrent encore 96,6\% des besoins en énergie domestique consommée par les ménages, en raison de la faible valorisation du potentiel représenté par l'énergie hydroélectrique (MEM, 2005).

L'utilisation du bois à des fins énergétiques domestiques nécessite une attention particulière si l'on veut assurer une gestion durable des écosystèmes forestiers qui le produisent. Comme l'affirmait Viers (1970), « la forêt et le développement ne font pas bon ménage jusqu'à maintenant, sauf pour quelques exceptions, les progrès de l'un s'accompagnent de la destruction de l'autre".

Selon Sanogo et al. (2006), l'usage du charbon de bois comme combustible principal pour la cuisine se répand de façon accélérée, au détriment du bois qui était encore le combustible presque exclusivement utilisé dans un passé récent. Cet usage du charbon de bois s'observe en grande partie dans toutes les grandes villes du Burundi.

Cette étude a pour objet de fournir des réponses aux interrogations suivantes: Quelle est la quantité annuelle de charbon utilisée par la population urbaine de Gitega et Bujumbura? Qu'en est-il pour toute la population urbaine du Burundi ? Quelle est la durabilité de ce système dans un pays aussi densément peuplé ? L'étude repose sur les hypothèses suivantes : la consommation de charbon de bois par la population urbaine contribue énormément à la perte de couvert forestier au Burundi ; les espaces forestiers des différentes provinces du Burundi sont inégalement exploités pour la production du charbon de bois; les essences forestières présentes au Burundi sont inégalement appréciées pour la carbonisation.

\section{Méthodes}

\section{Sites d'étude}

Cette étude a été réalisée dans deux des premières villes du Burundi : Bujumbura et Gitega. La ville de Bujumbura est située à l'ouest du Burundi, sur la frange côtière du nord du lac Tanganyika ( $3^{\circ} 22^{\prime} 32^{\prime \prime}$ de latitude Sud, 29²1' 33”' de longitude Est), à 787 m d'altitude. Elle compte 902000 habitants (ISTEEBU, 2015) et sa superficie ne cesse de s'agrandir. La ville de Gitega est située au centre du Burundi, à 102 km

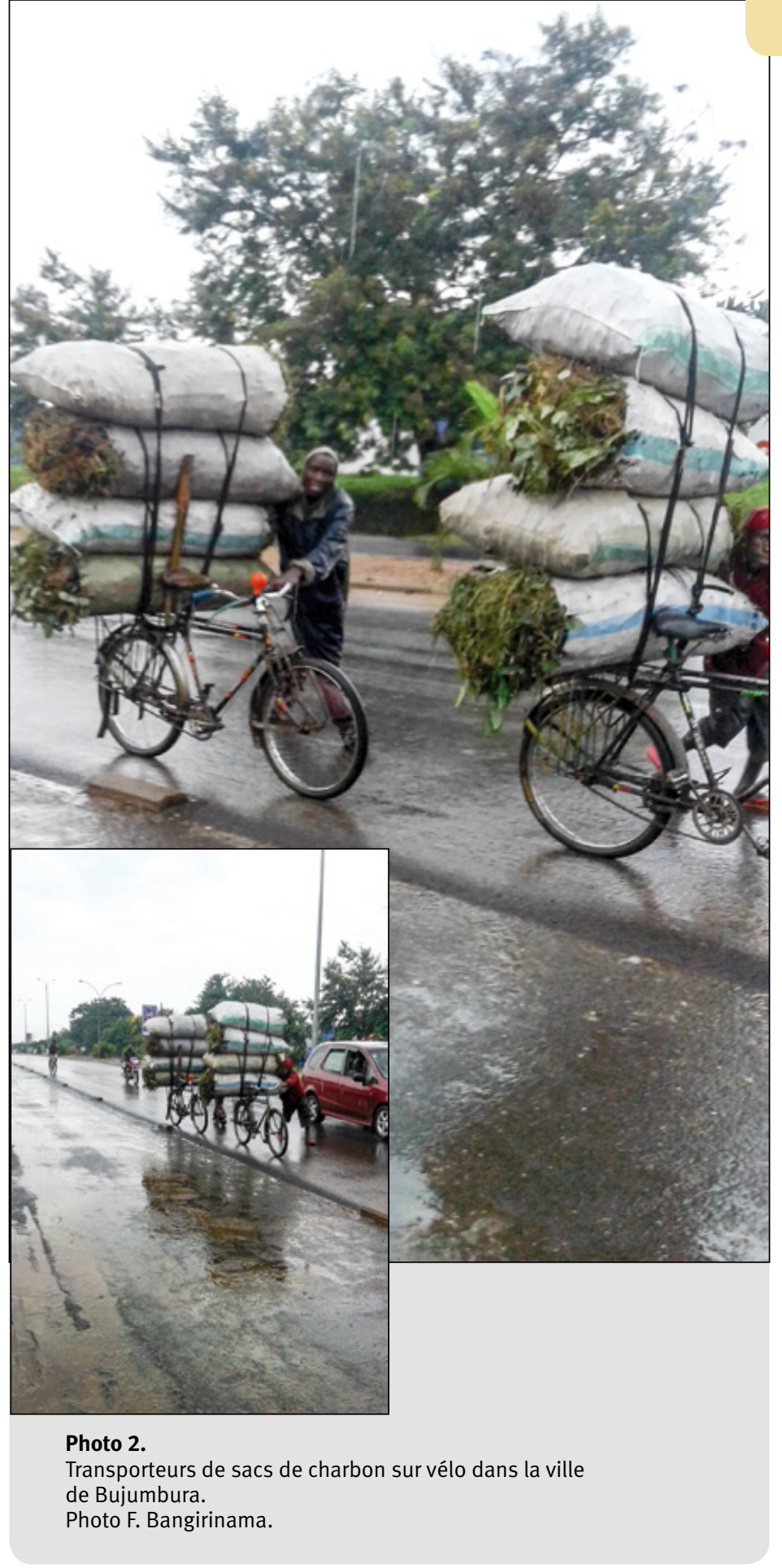

de Bujumbura, plus précisément dans la province de Gitega. Sa population avoisine 84000 habitants (ISTEEBU, 2015) et sa superficie est également en expansion.

\section{Collecte des données}

La collecte des données a été assurée d'avril à juillet 2014 à l'aide d'un questionnaire d'enquête. Les données recueillies concernaient la quantité de charbon de bois selon les différents types de transporteurs (camions et 


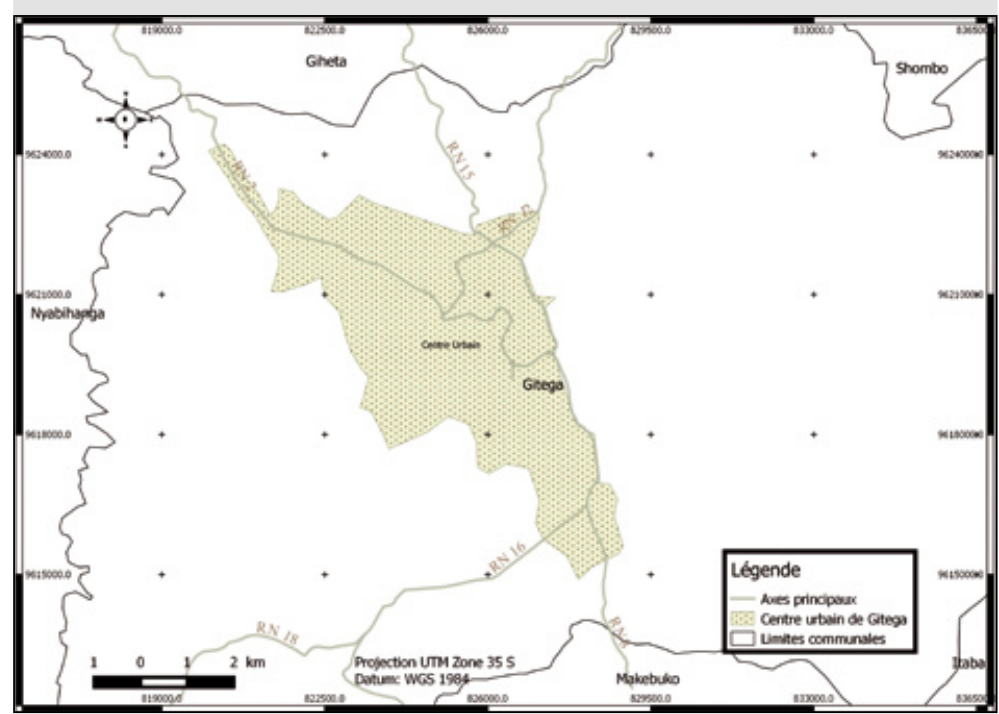

Figure 1.

Principaux axes routiers entrant dans la ville de Gitega (enquête réalisée sur trois axes routiers : Route nationale 2 (RN 2), Route nationale 8 (RN 8), Routes nationales 15 et 12 combinées (RN 15 et RN 12 combinées).

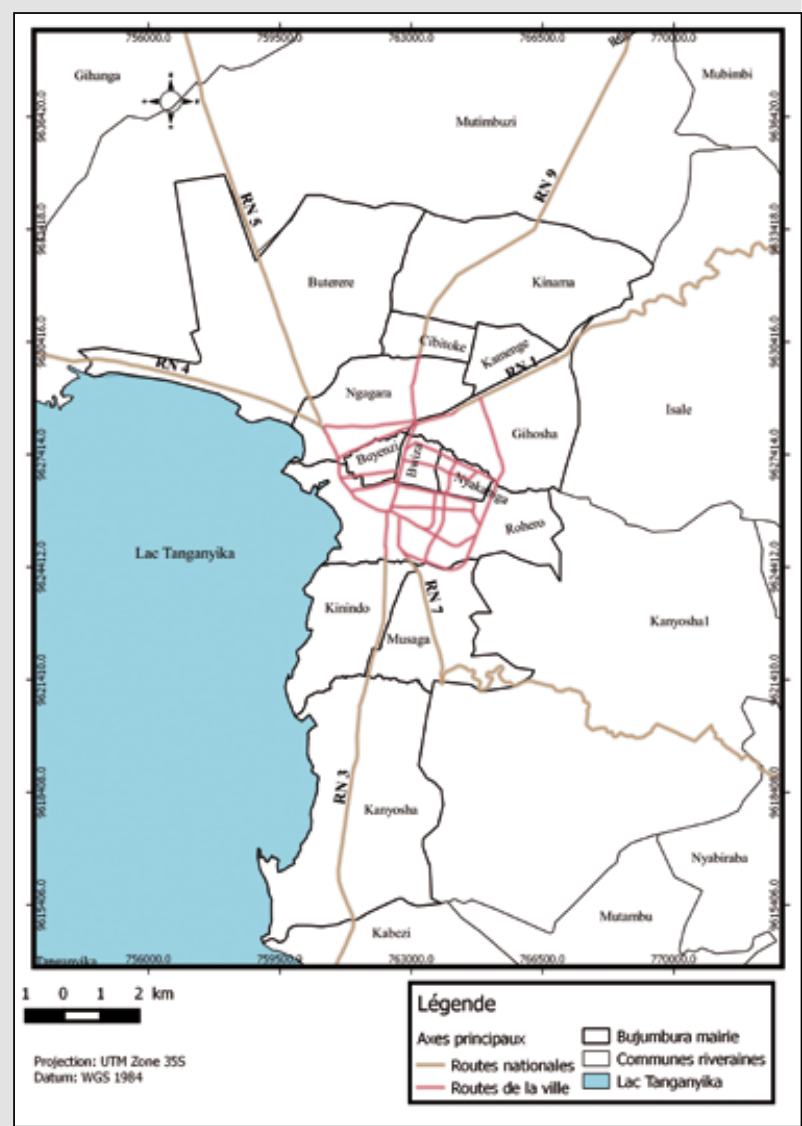

Figure 2.

Principaux axes routiers entrant dans la ville de Bujumbura (enquête réalisée sur quatre axes routiers : Route nationale 1 (RN 1) dans la zone de Kamenge, Route nationale 3 (RN 3) dans la zone de Kanyosha, Route nationale 7 (RN 7) dans la zone de Musaga et Route nationale 9 (RN 9) dans la zone de Kinama, entrée de Carama). vélos), la provenance du charbon de bois, le type d'essence utilisé dans la carbonisation, ainsi que les types de boisement exploités. Quatre axes routiers principaux (RN 1, RN 7, RN 9 et RN 3) menant vers la ville de Bujumbura (figure 1) et trois (RN 2, RN 8, RN 15 et RN 12 combinés) à Gitega (figure 2) ont été ciblés par l'enquête.

\section{Analyse des données}

Les sacs transportés par les transporteurs à vélo et les grossistes circulant en camion ont été pesés à l'aide d'une balance à suspension.

Après avoir collecté toutes les données sur le nombre de sacs enregistrés dans les deux villes pour les différents types de transporteurs, il a été déterminé la quantité moyenne de charbon hebdomadairement puis annuellement acheminée pour chaque type de transporteur.

L'eucalyptus étant l'essence la plus utilisée dans la confection du charbon de bois au Burundi, la superficie de plantations perdue annuellement a été déduite sur la base d'une productivité de 15 à 20 tonnes par hectare d'eucalyptus et par an (Boulier et Simon, 2009).

Enfin, à partir de la population urbaine des deux villes étudiées, il a été extrapolé l'impact global de la consommation de charbon de bois par la population urbaine totale du Burundi, qui s'élève à 1474000 habitants (ISTEEBU, 2015) (tableau I). La durabilité du système actuel de prélèvement et de valorisation du bois à des fins d'énergie domestique a été évaluée en estimant le nombre d'années durant lequel ce système peut être maintenu.

\section{Résultats}

\section{Ville de Bujumbura}

\section{Approvisionnement hebdomadaire}

Un total de 1987 sacs de charbon transportés à vélo est enregistré par semaine, soit l'équivalent de 89 tonnes de charbon de bois, avec une moyenne de $45 \mathrm{~kg}$ par sac. Un total de 12135 sacs de charbon de bois transportés par camion est comptabilisé chaque semaine, soit l'équivalent de 995 tonnes, avec une moyenne de $82 \mathrm{~kg}$ par sac. Dans les deux cas, les entrées de Kamenge (RN 1) et de Musaga (RN 7) représentent une quantité considérable par rapport aux autres.

Ainsi, la ville de Bujumbura reçoit chaque semaine 1084 tonnes de charbon de bois (tableau II).

\section{Provenance}

Les provinces ne fournissent pas de la même façon le charbon de bois. Le tableau III illustre la répartition de la quantité totale de charbon enregistrée dans la ville de Bujumbura suivant les provinces d'origine.

La province de Bururi vient en première position avec $44 \%$, puis la province de Mwaro (16\%), celle de Gitega (13\%) et celle de Makamba (11\%). Les autres provinces ont une contribution moins élevée.

Les provinces du Sud du pays (Bururi et Makamba) approvisionnent la ville de Bujumbura pour $55 \%$ de ses besoins en charbon de bois. Viennent ensuite celles du 
Tableau I.

Évolution de la population urbaine par province au Burundi (ISTEEBU, 2015)

(la province de Mwaro n'était pas encore créée en 1990).

\section{Province}

Population urbaine 1990

2945

894

Bujumbura

Bururi

Cankuzo

Cibitoke

Gitega

Karusi

Kayanza

Kirundo

Makamba

Muramvya

Muyinga

Mwaro

Ngozi

Rutana

Ruyigi

Bujumbura Mairie

Total
15816

1643

8280

20708

3403

6881

5181

5198

2290

5533

14511

1944

2377

235440

333044
Population urbaine 2008

20031

24347

45116

3624

23885

46308

10317

22580

14068

16183

9601

11755

3774

42835

11429

8847

497166

811866
Population urbaine 2015

36357

44191

81888

6578

43352

84051

18726

40984

25534

29373

17426

21336

6850

77747

20744

16058

902379

1473574

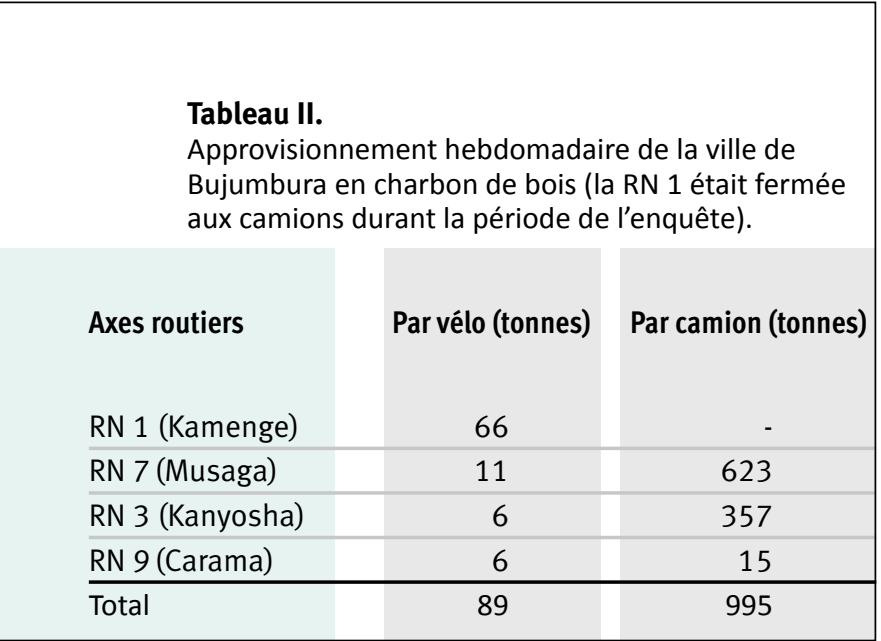

centre du pays (Gitega, Muramvya, Karusi, Mwaro) avec $40 \%$, puis celles du nord (Muyinga et Kayanza) avec $4 \%$. La contribution de celles de l'ouest du Burundi (Bubanza) est presque nulle $(0,2 \%)$.

L'eucalyptus est l'essence la plus employée dans la carbonisation, pour la totalité des enquêtés. Les autres espèces exploitées sont notamment Acacia mearnsii, Pericopsis angolensis et Grevillea robusta. Les boisements privés (98\% des enquêtés) sont beaucoup plus exploités que d'autres types de formations forestières.

Tableau III.

Provenance du charbon de bois utilisé dans la ville de Bujumbura.

\begin{tabular}{l|r|r|} 
Province & Quantité totale (tonnes) & $\%$ \\
\hline Bururi & 475 & 43,8 \\
\hline Mwaro & 172 & 15,9 \\
\hline Gitega & 145 & 13,4 \\
\hline Makamba & 114 & 10,5 \\
\hline Muramvya & 109 & 10,1 \\
\hline Kayanza & 25 & 2,3 \\
\hline Bujumbura rural & 22 & 2,0 \\
\hline Muyinga & 13 & 1,2 \\
\hline Karusi & 7 & 0,6 \\
\hline Bubanza & 2 & 0,2 \\
\hline Total & 1084 & 100 \\
\hline
\end{tabular}




\section{Ville de Gitega}

\section{Approvisionnement hebdomadaire}

Le nombre de sacs enregistrés par semaine est de 5411 , soit l'équivalent de 244 tonnes de charbon de bois. La RN 8 fournit presque la moitié (49\%) du charbon de bois consommé à Gitega.

Seuls 200 sacs de charbon de bois, soit l'équivalent de 16 tonnes, sont enregistrés par semaine pour les transports par camion. La plupart des transporteurs grossistes se dirigent vers la ville de Bujumbura plutôt que vers celle de Gitega.

La ville de Gitega consomme ainsi par semaine une quantité totale de 260 tonnes de charbon de bois (tableau IV).

\section{Provenance}

Le charbon de bois approvisionné dans la ville de Gitega provient pour $95 \%$ de la province de Gitega et pour $5 \%$ des provinces frontalières (tableau $\mathrm{V}$ ).

\section{Toutes les villes des chefs-lieux de province}

La consommation dans les deux villes est équivalente à environ 1344 tonnes de charbon de bois par semaine et 70100 tonnes par an. La consommation de la ville de Bujumbura est quatre fois plus élevée que celle de Gitega, soit respectivement $81 \%$ contre $19 \%$. En extrapolant pour

\section{Tableau IV.}

Approvisionnement hebdomadaire de la ville de Gitega en charbon de bois (aucun camion n'a été enregistré sur les axes RN 2 et RN 8).

\begin{tabular}{l|c|c|} 
Axes routiers & Par vélo (tonnes) & Par camion (tonnes) \\
\hline RN 2 & 41 & - \\
\hline RN 8 & 127 & - \\
\hline RN 12 et RN 15 \\
combinées
\end{tabular}

toutes les villes des chefs-lieux des provinces, la consommation de charbon de bois peut être estimée à 2008 tonnes par semaine et 104718 tonnes par an (tableau VI).

\section{Impact de la consommation de charbon de bois}

Les villes de Gitega et Bujumbura utilisant 70100 tonnes charbon de bois par an, dans l'hypothèse où cette quantité de charbon provient uniquement de l'eucalyptus (essence la plus employée pour la carbonisation) et en considérant que la productivité de cette dernière est de 15 à 20 tonnes de charbon par hectare, cette quantité de charbon proviendrait de la coupe annuelle d'une superficie de 3505 à 4673 ha.

La consommation de charbon de bois de toutes les villes des chefs-lieux des provinces entraînerait ainsi une coupe annuelle d'une superficie de 5236 à 6981 ha.

À ce rythme, la couverture forestière du Burundi, estimée à 171625 ha, disparaîtrait d’ici 25 à 33 ans.

\section{Tableau V.}

Provenance du charbon de bois utilisé dans la ville de Gitega.

\begin{tabular}{l|r|r|} 
Province & Quantité totale (tonnes) & \% \\
\hline Gitega & 248 & \\
\hline Muramvya & 10 & 95,5 \\
\hline Karusi & 2 & 3,9 \\
\hline Rutana & 0 & 0,6 \\
\hline Total & 260 & 0,0 \\
\hline
\end{tabular}

\section{Tableau VI.}

Estimation de la consommation annuelle de charbon de bois.

\begin{tabular}{l|r|r|r|}
\hline Villes & Population & Consommation hebdomadaire (tonnes) & Consommation annuelle (tonnes) \\
\hline Bujumbura Mairie & 902379 & 1084 & 56548 \\
\hline Gitega & 84051 & 260 & 13552 \\
\hline Bujumbura Mairie et Gitega & 986430 & 1344 & 700100 \\
\hline Toutes les villes du pays & 1473574 & 2008 & 104718 \\
\hline
\end{tabular}




\section{Discussion}

\section{Quantité totale de charbon de bois consommée}

Les résultats de l'enquête révèlent que respectivement 56548 tonnes et 13552 tonnes de charbon de bois sont consommées chaque année par la population urbaine de Bujumbura et de Gitega, soit 70100 tonnes de charbon de bois.

Dans la ville de Bujumbura, 29665 tonnes de charbon de bois ont été consommées en 1994 selon le MEM (1994, cité par Semwanga, 1996), 18857 tonnes l'ont été en 2011 selon le MEEATU (2011) et en 2013, ce sont 42742 tonnes de charbon qui ont été consommées selon Manirakiza (2013). Cet auteur avait seulement considéré deux axes routiers (RN 1 et RN 7), contre quatre (RN 1, RN 7, RN 9, RN 3) dans la présente étude.

Dans la ville de Gitega, 428 tonnes de charbon de bois ont été consommées en 2003 selon Gumiriza (1996). La ville est en pleine expansion, ce qui explique cette nette augmentation de la consommation de charbon, estimée actuellement à 13552 tonnes par an par notre enquête.

Une situation similaire est observée dans les villes des pays limitrophes. En République démocratique du Congo (RDC), la consommation de charbon de bois était respectivement estimée à 490000 tonnes et 16000 tonnes pour Kinshasa et Kisangani en 2010 (Schure et al., 2011). Au Rwanda, la capitale Kigali joue un rôle dominant dans l'exploitation du bois à des fins énergétiques, avec une demande en charbon de bois qui représente quelque $60 \%$ des besoins nationaux. En 2009, la consommation de charbon de bois était ainsi de 132000 tonnes pour la ville de Kigali (Drigo et al., 2013).

Au Burundi, une consommation annuelle de 104718 tonnes de charbon de bois par la population urbaine a été estimée par la présente étude, contre 42997 tonnes de charbon de bois sur la période 1994-1998 (Nkurunziza, 1999).

Ces résultats confirment l'effet de la croissance démographique et, par conséquent, de l'urbanisation sur la consommation de charbon de bois, non seulement par la population des grandes villes, mais aussi par toute la population des centres urbains secondaires. En effet, le taux de corrélation est de 0,93 entre la courbe d'augmentation de la population urbaine et celle de la consommation de charbon de bois entre 1990 et 2015 (figure 3) (MININTER, 2008 ; MEM, 2011b, 2012 ; Ruzima et al., 2012).

\section{Provenance du charbon de bois}

Le charbon de bois consommé dans la ville de Bujumbura provient pour $55 \%$ des régions du Sud-Est du pays, et celui consommé dans la ville de Gitega pour $95 \%$ de la province de Gitega. Ces résultats sont semblables à ceux du MEEATU (2011), selon lesquels les régions du sudest du pays venaient en première position (57\%) pour la fourniture du charbon de bois à la ville de Bujumbura. Ils correspondent également à ceux de Manirakiza (2013), qui montrent que les régions du sud-est du pays fournissent $50 \%$ du charbon de bois, mais aussi à ceux de Gumiriza
(1996), d'après lesquels le charbon de bois approvisionné par la ville de Gitega provient pour $93 \%$ des zones et communes proches, et pour $7 \%$ des provinces frontalières comme celle de Karusi.

\section{Essences utilisées dans la production de charbon de bois}

L'eucalyptus (Eucalyptus spp.) est l'essence forestière la plus appréciée pour la production du charbon de bois, comme $100 \%$ des enquêtés l'ont affirmé sur le terrain. La dominance de l'eucalyptus dans les boisements privés du pays est liée à sa vitesse de croissance élevée par rapport aux autres essences (Pinus, Grevillea, Acacia). Ce constat est également celui de l'enquête menée par le MEEATU (2011), qui indique que cette essence intervient pour $96 \%$ dans la fabrication du charbon de bois.

\section{Types de boisements exploités dans la production de charbon de bois}

L'enquête confirme que le charbon de bois provient en grande partie des boisements privés ( $98 \%$ des enquêtés). De plus, la majorité des enquêtés précisent qu'ils obtiennent difficilement leur charbon de bois et doivent parcourir de longues distances pour s'en procurer. La plupart du charbon de bois consommé dans la partie urbaine de Bujumbura provient des provinces les plus éloignées, alors que celles qui sont proches n'en fournissent plus qu'une quantité minime.

Le constat est que les écosystèmes forestiers situés en dehors des aires protégées sont en cours de disparition. Ils ont déjà disparu dans les provinces limitrophes de la ville de Bujumbura et diminuent de jour en jour dans les provinces lointaines comme Bururi, Gitega, Karuzi et Makamba. Le même constat a été déjà formulé par le MEEATU (2012).

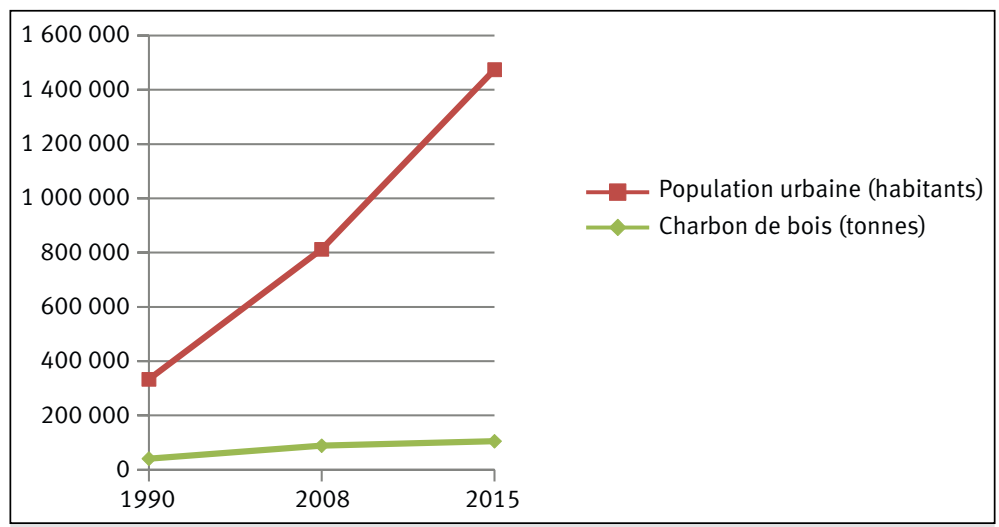

Figure 3.

Évolution de la population urbaine et de la consommation de charbon de bois au Burundi entre 1990 à 2015 (la corrélation entre les deux séries de données étant de 0,93). 


\section{Pertes de couvert forestier}

La consommation de charbon de bois par la population des villes de Gitega et Bujumbura entraîne chaque année une perte équivalente de 3505 à 4673 ha de couvert forestier, et la population urbaine totale du pays générerait une perte annuelle comprise entre 5236 et 6980 ha. Manirakiza (2013) a estimé que la consommation de charbon de bois de la population de Bujumbura se traduisait par une perte annuelle de 2442 ha de forêt, superficie cependant sous-estimée étant donné que son enquête n'avait concerné que deux axes routiers (RN 1 et RN 7), contre quatre pour la présente étude (RN 1, RN 7, RN 9 et RN 3).

Cette perte annuelle de couvert forestier est alarmante dans un pays où la superficie forestière est estimée à 171625 ha (Bararwandika et al., 2012). À ce rythme, les 171625 ha actuels seront décimés dans 25 à 33 ans.
De même, l'augmentation de la demande urbaine en bois énergie se traduit par une pression accrue sur les ressources forestières de la RDC, ce qui est particulièrement problématique en l'absence de mesures incitatives pour la restauration ou la gestion durable de cette ressource (Schure et al., 2011).

Pour pallier ce problème, il faudrait planter au moins les 5984 ha perdus annuellement pour satisfaire la consommation urbaine. Or, le Burundi ne dispose plus d'espace suffisant pour mettre en place des programmes de reboisement à grande échelle (Nduwamungu, 2011). Selon Koyo (2004), les seules zones disponibles pour ce genre de plantations sont les rares sommets de colline, l'autre moyen d'accroître le couvert forestier étant de combiner les arbres aux cultures vivrières et de créer des plantations en ligne. La plantation des arbres le long des axes routiers constitue une autre alternative durable.

Pour les plantations disponibles, la régénération naturelle assistée pour permettre l'accroissement de la productivité constitue une voie possible. Cette technique a été adaptée et testée par le projet Makala dans le bassin d'approvisionnement en bois de feu de Kinshasa, pour améliorer les systèmes de culture sur abattis-brûlis et contribuer à l'enrichissement des jachères forestières (Peltier et al., 2014).

La recherche d'espèces ou de variétés mieux adaptées et plus rentables, mais aussi de pratiques plus appropriées, pourrait constituer une autre voie. À Madagascar, Eucalyptus robusta a été retenu comme espèce de reboisement et intégré au monde rural grâce à sa remarquable capacité d'adaptation (Verhaegen et al., 2014).

II faudrait en outre rehausser le taux d'utilisation des énergies renouvelables (biogaz, énergie solaire, énergie éolienne) pour diminuer le plus possible l'utilisation du charbon de bois. Les nouvelles techniques de fabrication de charbon à base de déchets (briquettes) pourraient permettre l'atténuation de la situation actuelle. Plusieurs pays (Ouganda, Kenya, Malawi, Rwanda, Éthiopie, Haïti, Tanzanie, Mali) ont déjà mené des études sur des briquettes combustibles confectionnées à partir des déchets solides (Dusabe, 2013). Une entreprise de fabrication de ces briquettes (Burundi Quality Stoves) est déjà fonctionnelle au Burundi. En Ouganda, au moins $5 \%$ de la consommation de bois de chauffage et $50 \%$ de celle de charbon de bois peuvent être remplacés par des briquettes combustibles à base de déchets (Ferguson, 2012).

Enfin, l'amélioration de l'efficience énergétique est une nécessité absolue pour un avenir plus proche, en favorisant des techniques plus rentables de carbonisation et d'utilisation du charbon de bois. Le potentiel et les techniques des foyers améliorés dans les pays en développement pourraient contribuer à une exploitation plus durable de cette ressource naturelle de base (Schure et al., 2011).

Plantation d'eucalyptus dans la commune de Bukirasazi, province de Gitega.

Photo F. Bangirinama. 


\section{Références bibliographiques}

Aruna Sefu J., 2011. La consommation du combustible braise (charbon de bois) par les ménages de Bukavu et son impact sur la déforestation au Sud-Kivu. Mémoire, ISDR Bukavu, République démocratique du Congo, 125 p.

Bararwandika A., Ndereyimana E., Barindogo V., Ntakarutimana O., 2012. Évaluation des ressources forestières mondiales. Burundi. Rapport national. Rome, Italie, FAO, Département des forêts, $158 \mathrm{p}$.

Boulier J., Simon L., 2009. Atlas des forêts dans le monde. Protéger, développer, gérer une ressource vitale. Paris, France, Éditions Autrement, 80 p.

Drigo R., Munvehirwe A., Nzabanita V., Munyampundu A., 2013. Final report Update and upgrade of WISDOM Rwanda and Woodfuels value chain analysis as a basis for the Rwanda Supply Master Plan for fuelwood and charcoal. Florence, Italie, Agriconsulting S.p.A., 173 p.

Dusabe M. S., 2013. Étude de faisabilité technique et financière de la valorisation des déchets ménagers organiques, papiers et cartons pour la fabrication des briquettes combustibles à Bujumbura, Burundi. Mémoire de master en ingénierie de l'eau et de l'environnement, Institut international d’ingénierie, Burkina Faso, 64 p.

Ferguson H., 2012. Briquette businesses in Ouganda: The potential for briquette enterprises to address the sustainability of the Ugandan biomass fuel market. Londres, Royaume-Uni, GVEP International.

Gumiriza T., 1996. La consommation du bois-énergie dans la ville de Gitega. Mémoire de géographie, Université du Burundi, $86 \mathrm{p}$.

ISTEEBU, 2015. Estimations de la population urbaine des villes des chefs-lieux des provinces du Burundi. Document non publié.

Koyo J. P., 2004. Watershed management case study: Burundi. Comprehensive, integrated watershed and swamp management. Rome, Italie, FAO.

Manirakiza D., 2013. Effets de la consommation du charbon de bois sur la dégradation de l'environnement : cas de la ville de Bujumbura. CURDES, Université du Burundi, FSEA, Rapport provisoire, $11 \mathrm{p}$.

MEEATU, 2011. Rapport de l'enquête sur le flux d'approvisionnement en bois énergie de Bujumbura. IFDC, Département des forêts, $60 \mathrm{p}$.

MEEATU, 2012. Rapport sur l'état des ressources génétiques forestières au Burundi. Bujumbura, Burundi, Département des forêts, $54 \mathrm{p}$.

MEM, 2005. Bilan énergétique national. Rapport annuel. Bujumbura, Burundi, $55 \mathrm{p}$.

MEM, 2011a. Lettre de politique énergétique. Bujumbura, Burundi, DGEE, $35 \mathrm{p}$.

MEM, 2011b. Élaboration de la Stratégie sectorielle pour le secteur de l'énergie au Burundi. Bujumbura, Burundi, DGEE, $149 \mathrm{p}$.
MEM, 2012. Lettre de politique énergétique. Bujumbura, Burundi, DGEE, 35 p.

MININTER, 2008. Recensement général de la population et de l'habitat du Burundi. Volume 3 : Analyse. Tome 6 : État et structures de la population. Bujumbura, Burundi, $88 \mathrm{p}$.

Nduwamungu J., 2011. Plantations forestières et îlots boisés au Rwanda. Nairobi, Kenya, African Forest Forum, 109 p.

Nkurunziza F., 1999. Rapport d'étude sur les données du bois-énergie au Burundi. FAO, UE, BAD, 125 p.

Peltier R., Dubiez E., Diowo S., Gigaud M., Marien J.-N., Marquant B., Peroches A., Proces P., Vermeulen C., 2014. Assisted Natural Regeneration in slash-and-burn agriculture: Results in the Democratic Republic of the Congo. Bois et Forêts des Tropiques, 321 (3) : 79-67. http://bft.cirad.fr/ $\mathrm{cd} / \mathrm{BFT}$ 79-67 321.pdf

Ruzima S., Nintunze A., Nyamuhwata S., 2012. Situation des infrastructures au Burundi. Synthèse du Rapport final. Bujumbura, Burundi, Ministère des Transports, des Travaux publics et de l’Équipement, Direction générale de la cordination des équipements, $25 \mathrm{p}$.

Sanogo C. A., Elhadji Mahamane M. L., Khennas S., Konandji H., Van der Plas J. R., Girard P., 2006. Techniques améliorées de carbonisation au Sahel. PREDAS et UE, coll. Les guides techniques du PREDAS, $38 \mathrm{p}$.

Schure J., Ingram V., Akalakou-Mayimba C., 2011. Bois énergie en RDC : Analyse de la filière des villes de Kinshasa et de Kisangani. Projet Makala/Cifor, 88 p.

Semwanga O., 1996. Consommation du charbon de bois par les ménages de la mairie de Bujumbura et ses conséquences environnementales et économiques. Mémoire, Université du Burundi, FACAGRO, 88 p.

Verhaegen D., Randrianjafy H., Rakotondraoelina Andriatsitohaina H., Trendelenburg Rakotonirina M.-C., Andriamampianina N., Montagne P., R, et al., 2014. Eucalyptus robusta pour une production durable de bois énergie à Madagascar : bilan des connaissances et perspectives. Bois et Forêts des Tropiques, 320 (2) : 30-15. http://bft.cirad.fr/ cd/BFT 30-15 320.pdf

Viers G., 1970. Géographie des forêts. Paris, France, PUF, $222 \mathrm{p}$. 